\title{
Modal Fields Calculation Using the Finite Difference Beam Propagation Method
}

\author{
Frank Wijnands, Hugo J. W. M. Hoekstra, Gijs J. M. Krijnen, Member, IEEE, and René M. de Ridder
}

\begin{abstract}
A method is described to construct modal fields for an arbitrary one- or two-dimensional refractive index structure. An arbitrary starting field is propagated along a complex axis using the slowly varying envelope approximation (SVEA). By choosing suitable values for the step-size, one mode is maximally increased in amplitude on propagating, until convergence has been obtained. For the calculation of the next mode, the mode just found is filtered out, and the procedure starts again. The method is tested for one-dimensional refractive index structures, both for nonabsorbing and for absorbing structures, and is shown to give fast convergence.
\end{abstract}

\section{INTRODUCTION}

B EAM Propagation Methods (BPM's) are very powerful to simulate the propagation of light in structures which cannot be treated analytically. Two frequently used BPM's are the Fourier Transform BPM (FTBPM) [1]-[3] and the Finite Difference BPM (FDBPM) [4]-[6]. There are various methods to perform a modal analysis for an arbitrary refractive index structure. Two-dimensional transfer matrix methods can be used for two-dimensional structures [7]-[10], whereas threedimensional structures require other methods. One method comes down to solving an eigenvalue problem with the dimension of the matrix to be diagonalized being equal to the number of grid points of the cross section [11]. Another method, by Yevick and Hermansson [12], consists of propagating a field distribution along an imaginary propagation direction (throughout this paper, the propagation direction is along the (in general complex) $z$-axis). The idea is that the guided mode with the highest effective index gets the maximum amplitude increase during propagation. They use as propagation scheme the FTBPM.

It is the aim of this paper to show that the FDBPM is quite suitable for solving the modal fields equation. The advantage of the FDBPM is its fast convergence. For the FDBPM the matrix problem can be solved such that selected guided modes can be blown up, as will be shown in this paper. To do so, one may need a large propagation step, which is allowed in the FDBPM. Hence the number of propagation steps in order to blow up a selected mode is in general much smaller for the FDBPM than for the FTBPM. Moreover, the FDBPM gives much more accurate results for large index contrasts (see, e.g., [13]-[15]).

Manuscript received August 31, 1994.

F. Wijnands, H. J. W. M. Hoekstra, and R. M. de Ridder are with the MESA Research Institute, University of Twente, 7500 AE Enschede, the Netherlands

G. J. M. Krijnen is with the University of Central Florida Center for Research in Electro-Optics and Lasers, Orlando FL 32826 USA.

IEEE Log Number 9406458.
The waveguide structure to be analyzed is in general $z$ dependent. Since we are interested in finding the modal fields at a cross section of the structure for a given value of $z$, the given cross section will be extended in the direction of propagation, leading to a $z$-invariant "calculation structure."

The method is tested for one-dimensional refractive index structures. The method can be easily generalized to twodimensional refractive index structures. The only difference is that the matrix problem is not a tridiagonal matrix problem anymore in that case and hence a different matrix solver should be used than in the one-dimensional case. An efficient iterative matrix solver method is the (preconditioned) conjugate gradient method [16]. Another, noniterative, matrix solution method is the Alternating Direction Implicit method [17], [18]. A combination of these two methods gives a very efficient algorithm, of which the results will be published elsewhere [19]. The paper is organized as follows. In Section II the method is described, both for nonabsorbing and for absorbing structures. Results for some one-dimensional structures are presented in Section III. A discussion of the results follows in Section IV.

\section{THE METHOD}

\section{A. Propagation Along a Real Axis with FDBPM}

In this paper the two-dimensional case is treated. Assuming TE-polarization and a time dependence term $e^{i \omega t}$, we have

$$
\nabla^{2} E(x, z)+k_{0}^{2} n^{2}(x, z) E(x, z)=0 .
$$

A scalar field distribution $E(x, z)$ can be written, according to the slowly varying envelope approximation (SVEA), as

$$
E(x, z) \equiv \psi(x, z) e^{-i k_{0} n_{0} z}
$$

where $n_{0}$ is a suitably chosen mean refractive index. Substituting (2) into (1) yields

$$
2 i k_{0} n_{0} \frac{\partial \psi}{\partial z}=\left[\frac{\partial^{2}}{\partial x^{2}}+k_{0}^{2}\left(n^{2}(x, z)-n_{0}^{2}\right)\right] \psi+\frac{\partial^{2} \psi}{\partial z^{2}} .
$$

Now $\psi$ is assumed to vary slowly as function of $z$ (see (2)). Hence the term $\partial^{2} \psi / \partial z^{2}$ in (3) can be neglected. Now discretize the field, $\psi_{j}^{s} \equiv \psi(s \Delta z, j \Delta x), j=1, \cdots, N$ and introduce the vector $\Psi^{s},\left(\Psi^{s}\right)_{j} \equiv \psi_{j}^{s}$. Then (3) is integrated for the discretized field, using the Crank-Nicolson scheme. The result is [5]:

$$
2 i k_{0} n_{0}\left(\boldsymbol{\Psi}^{s+1}-\Psi^{s}\right)=\boldsymbol{M}\left(\Psi^{s+1}+\boldsymbol{\Psi}^{s}\right) \frac{\Delta z}{2} .
$$


Here $\boldsymbol{M}$ is a tridiagonal matrix with $\boldsymbol{M}_{i j}=\frac{1}{(\Delta x)^{2}}, M_{i i}=$ $\frac{-2}{(\Delta x)^{2}}+k_{0}^{2}\left(n_{i}^{2}-n_{0}^{2}\right), 1 \leq i \leq N, j=i \pm 1, M_{i j}=0$ elsewhere. Transparent boundary conditions are used [20], and efficient interface conditions are applied [14]. For convenience, (4) is rewritten in the form

$$
\left[\mathbf{1}+i \boldsymbol{M} \Delta z /\left(4 k_{0} n_{0}\right)\right] \boldsymbol{\Psi}^{s+1}=\left[\mathbf{1}-i \boldsymbol{M} \Delta z /\left(4 k_{0} n_{0}\right)\right] \boldsymbol{\Psi}^{s} .
$$

Here 1 is the identity matrix. Following the analysis described in [15], suppose that the propagating field consists of a single guided mode $\boldsymbol{\Theta}^{s}$ with effective index $n_{e}$ corresponding to the discretized structure. Then $\Theta^{s}$ satisfies

$$
\mathbf{M} \Theta^{s}=k_{0}^{2}\left(n_{e}^{2}-n_{0}^{2}\right) \Theta^{s} .
$$

Substitution of (6) in (5) yields

$$
\Theta^{s+1}=\frac{4 \beta_{0}-i \Delta z\left(\beta_{e}^{2}-\beta_{0}^{2}\right)}{4 \beta_{0}+i \Delta z\left(\beta_{e}^{2}-\beta_{0}^{2}\right)} \Theta^{s}
$$

with $\beta_{e} \equiv k_{0} n_{e}, \beta_{0} \equiv k_{0} n_{0}$. If $m$ guided modes are present, say

$$
\Psi^{s}=\sum_{j=0}^{m-1} \alpha_{j} \Theta_{j}^{s}
$$

each with propagation constant $\beta_{e_{j}}$, then (7) is replaced by

$$
\begin{aligned}
\Psi^{s+1} & =\sum_{j=0}^{m-1} \frac{4 \beta_{0}-i \Delta z\left(\beta_{e_{j}}^{2}-\beta_{0}^{2}\right)}{4 \beta_{0}+i \Delta z\left(\beta_{e_{j}}^{2}-\beta_{0}^{2}\right)} \alpha_{j} \Theta_{j}^{s} \\
& \equiv \sum_{j=0}^{m-1} K_{j} \alpha_{j} \Theta_{j}^{s} .
\end{aligned}
$$

\section{B. Modal Field Solution Using FDBPM}

Consider a starting field distribution consisting of guided modes given by (8) and radiation modes. Our task is to find the constituent $m$ modal fields $\boldsymbol{\Theta}_{0}^{s}, \cdots, \boldsymbol{\Theta}_{m-1}^{s}$. In order to find the mode $\Theta_{j}^{s}$ (with, in general complex, propagation constant $\beta_{e_{j}}$ ), the idea is to choose $\Delta z$ such that $4 \beta_{0}+i \Delta z\left(\beta_{e_{j}}^{2}-\beta_{0}^{2}\right) \approx 0$. Fig. 1 shows $K_{0}, K_{1}$, defined in (9), as function of $\Delta z=$ ir $r \in \mathbf{R}^{+}$, for a bimodal nonabsorbing waveguide (Test 1 in Section III). From Fig. 1 one sees how the values for $\Delta z$ should be chosen in order to increase the relative amplitude of a certain mode. The method used to find all guided modes is based on the inverse iteration method [17]. It works as follows. For convenience, (4) is rewritten as

$$
\left[\boldsymbol{M}-i \frac{4 \beta_{0}}{\Delta z}\right] \boldsymbol{\Psi}^{s+1}=\left[-\boldsymbol{M}-i \frac{4 \beta_{0}}{\Delta z}\right] \boldsymbol{\Psi}^{s} \equiv \boldsymbol{f}^{s}
$$

for the sth iteration step. Now suppose one wants to find the complex eigenvector $\boldsymbol{\Theta}_{j}$ and the corresponding complex eigenvalue $\tau \equiv\left(\beta_{e_{j}}^{2}-\beta_{0}^{2}\right)$ of the matrix $\boldsymbol{M}$. The search may be limited to a certain region of $\beta_{e_{j}}$-values by choosing $\Delta z$ and $\beta_{0}$ such that the corresponding eigenvectors will blow up. E.g., for real refractive index profiles, if we put a lower bound on the possible effective indexes of the guided modes, one can choose $n_{0}$ equal to that bound. Only modal fields with $\beta_{e_{j}}-\beta_{0}>0$ will then satisfy $\left|\Theta^{s+1}\right|>\left|\Theta^{s}\right|$. If we limit ourselves to nonabsorbing and weakly absorbing structures

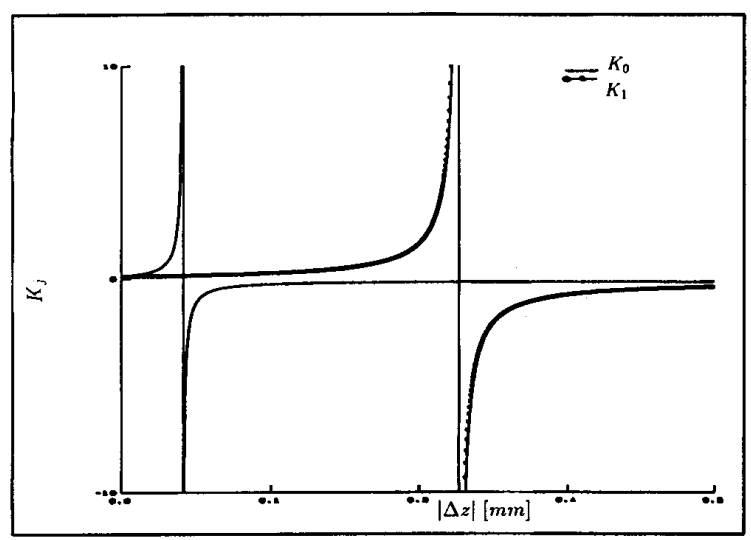

Fig. 1. Function $K_{j}(|\Delta z|), j=0,1$ for two modal fields of bimodal waveguide described in text and $\Delta z$ purely imaginary. Here $n_{0}$ was taken equal to 1.59 .

only, then for modal fields with complex effective indexes close to the real axis, the criterion is safe as well.

The value for $\Delta z$ is found from $i 4 \beta_{0} / \Delta z=\tau_{s}, s \geq 0$. Here $\tau_{0}$ should be estimated, and $\tau_{s}, s \geq 1$, can be calculated as described below. After having applied (10), for the next iteration step $\tau_{s}$ is replaced by

$$
\tau_{s+1}=\tau_{s}+\frac{\left.<\boldsymbol{f}^{s}, \boldsymbol{f}^{s}\right\rangle}{\left.<\mathbf{\Psi}^{\mathbf{s}+1}, \boldsymbol{f}^{\mathbf{s}}\right\rangle}
$$

with inner product $\langle\boldsymbol{\Omega}, \boldsymbol{\Lambda}\rangle \equiv \sum_{j=1}^{N}\left[\Omega_{j}\left(\Lambda_{j}\right)^{*}\right], N$ being the number of grid points. This change of eigenvalue is established by changing $\Delta z$ such that $i 4 \beta_{0} / \Delta z=\tau_{s+1}$. The approximate propagation constant $\beta_{e_{j}, s}$ is determined from the relation $\beta_{e_{j}, s}=\sqrt{\tau_{s}+\beta_{0}^{2}}$. In addition, $\Psi^{\mathbf{s}}$ is replaced by $\Psi^{\mathbf{s}+1}$ (which is renormalized first).

The motivation for this choice can be found in [17], and is briefly recalled below. Suppose one tries to solve the matrix problem

$$
\left[\boldsymbol{M}-\tau_{s}\right] \boldsymbol{\Psi}^{s+1}=\left[-\boldsymbol{M}-\tau_{s} \boldsymbol{\Psi}\right] \boldsymbol{\Psi}^{s}
$$

for given $\Psi^{s}$ and $\tau_{s}$ such that $\tau_{s}$ is near one of the eigenvalues, say $\tau$. Write $\Psi^{s}$ and $\Psi^{s+1}$ in terms of the modal fields as

$$
\boldsymbol{\Psi}^{s+1}=\sum_{k=0}^{m-1} \gamma_{k} \boldsymbol{\Theta}_{k}, \boldsymbol{\Psi}^{s}=\sum_{k=0}^{m-1} \delta_{k} \Theta_{k}
$$

with $\Theta_{k}$ having eigenvalue $\tau_{k}$. Inserting (13) to (12) gives

$$
\gamma_{k}=-\frac{\delta_{k}\left(\tau_{k}+\tau_{s}\right)}{\left(\tau_{k}-\tau_{s}\right)}, \quad 0 \leq k<m
$$

For the next iteration step, write $\boldsymbol{\Psi}^{s+2}=\sum_{k=0}^{m-1} \gamma_{k}^{\prime} \boldsymbol{\Theta}_{k}$. Then

$$
\gamma_{k}^{\prime}=\frac{\delta_{k}\left(\tau_{k}+\tau_{s+1}\right)\left(\tau_{k}+\tau_{s}\right)}{\left(\tau_{k}-\tau_{s+1}\right)\left(\tau_{k}-\tau_{s}\right)}, \quad 0 \leq k<m .
$$

Now if $\tau_{s}, \tau_{s+1} \approx \tau$, then by comparing (14) and (15) one sees that $\Psi^{s+2}$ is a better approximation of the eigenvector $\Theta_{j}$ than $\Psi^{s+1}$.

The choice for $\tau_{s+1}$ in (11) is based on the following argument. If $\Psi^{s+1} \approx \Theta_{j}$, then $\mathbf{M} \Psi^{s+1} \approx \tau \Psi^{s+1}$. Next, $\tau_{s+1}$ should be a better approximation of $\tau$ than $\tau_{s}$. Hence (12) can 


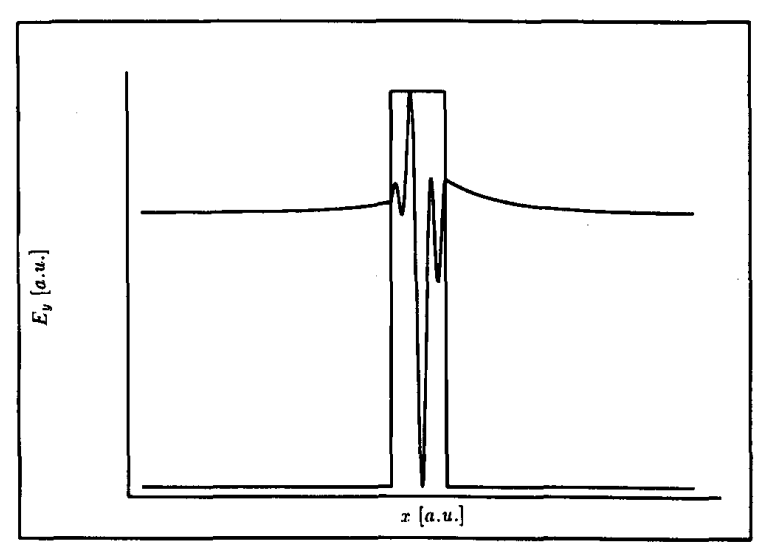

(a)

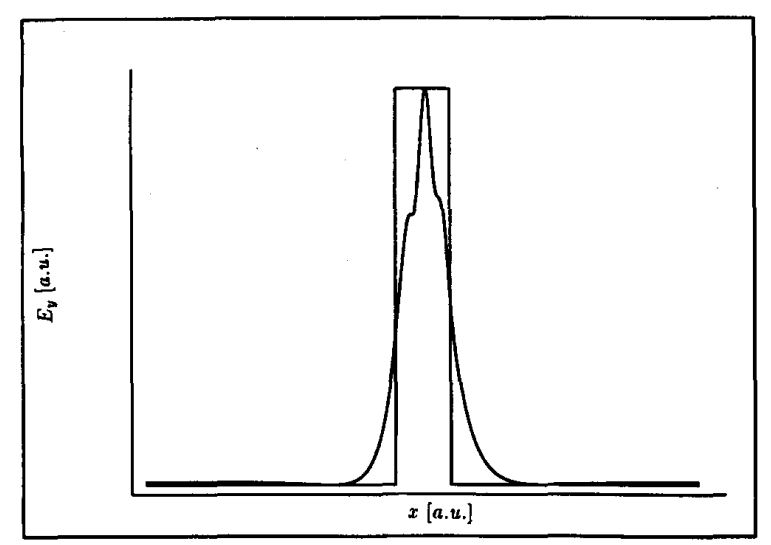

(c)

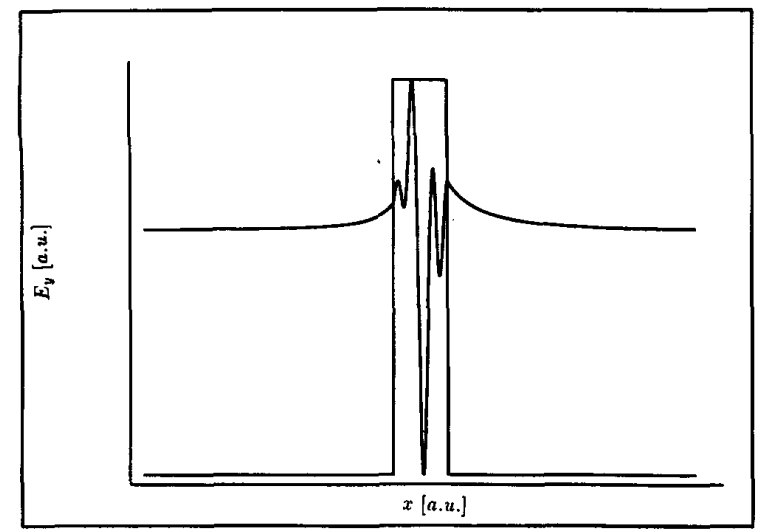

(e)

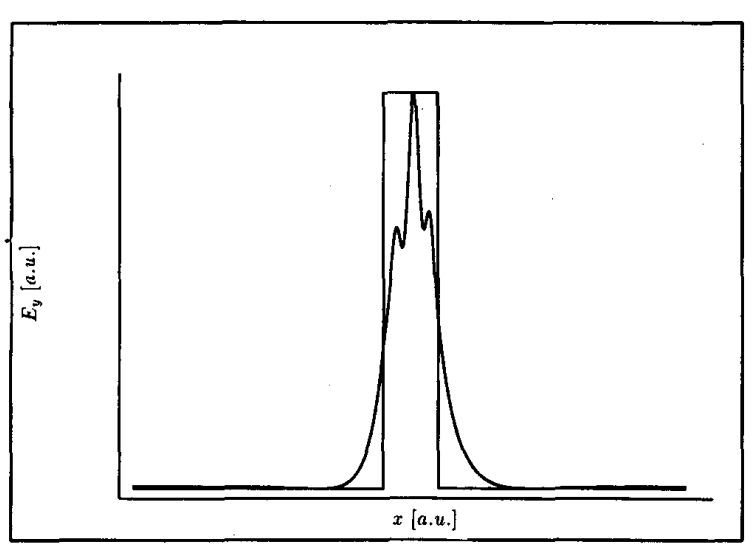

(b)

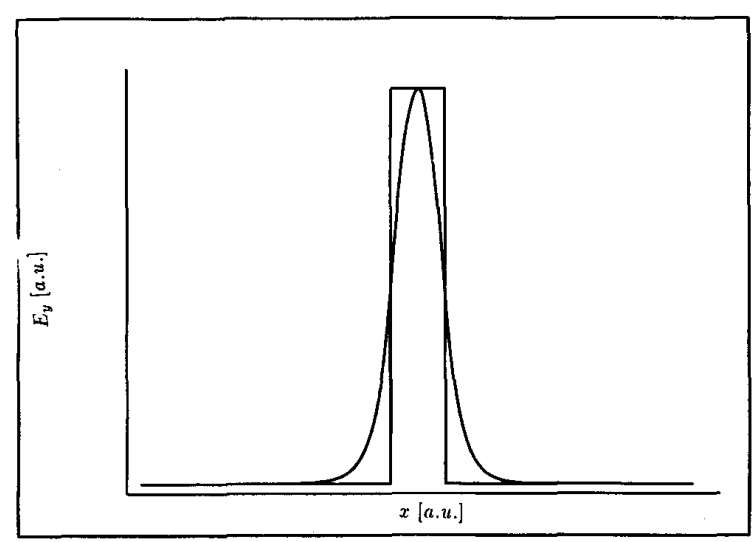

(d)

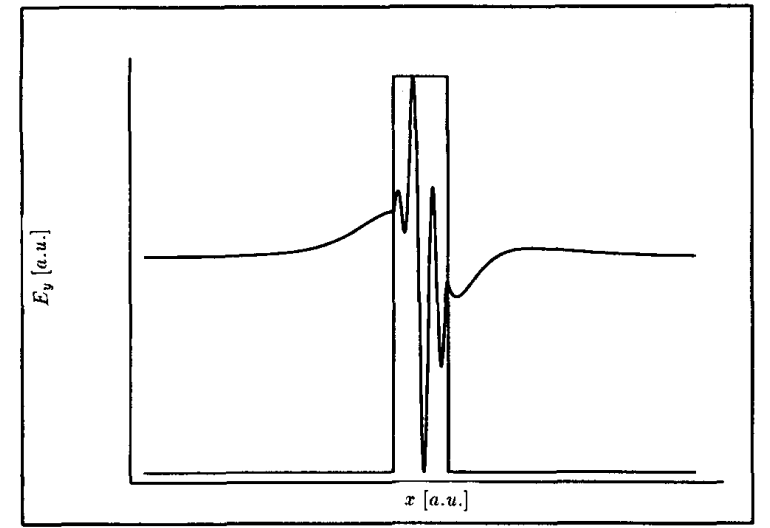

(f)

Fig. 2. Field profiles $E_{y}$ as function of $x$ after some subsequent propagation steps for bimodal single waveguide structure described in text (Section III, Test 1). The starting field, shown in (a), is a superposition of five sine and cosine functions, in combination with an appropriate exponentially decaying function. In (d), the $\mathrm{TE}_{0}$ mode has converged. In (e), the $\mathrm{TE}_{0}$ mode has been filtered out of the starting field, shown in (a), according to (16).

be approximated as $\left(\tau_{s+1}-\tau_{s}\right) \Psi^{s+1}=f^{s}$. By multiplying both sides with $\left(f^{s}\right)^{*},(11)$ is recovered.

Using this procedure, a sequence of values for $\beta_{e_{j}}$ is achieved, which eventually converges.

Now that a mode, denoted by $\Theta_{j}$, has been found, the next guided mode, if present, is sought. First construct a new starting field $\Psi^{0 \prime}$, orthogonal to $\Theta_{j}$, defined as

$$
\Psi^{0 \prime}=\Psi^{0}-\Theta_{j} \frac{<\Psi^{0}, \Theta_{j}>}{<\Theta_{j}, \Theta_{j}>} .
$$

The next problem is to choose a new trial value for $\Delta z$. If the value for $\Delta z$ is left unchanged, then the new starting 


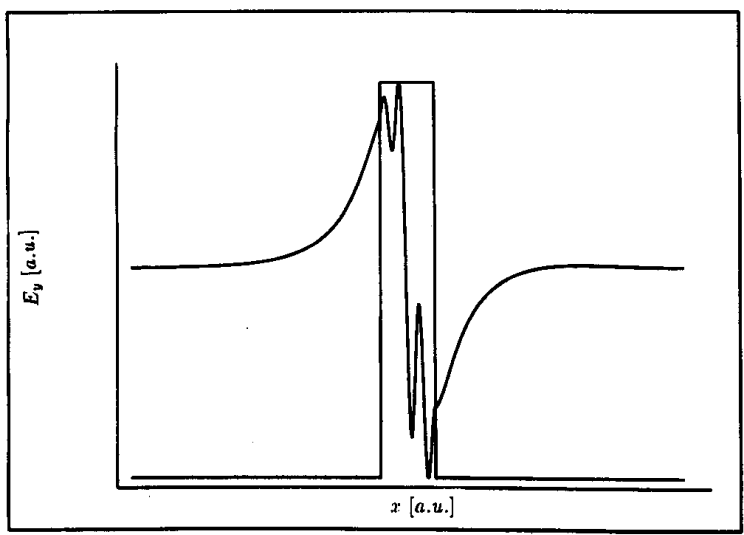

(g)

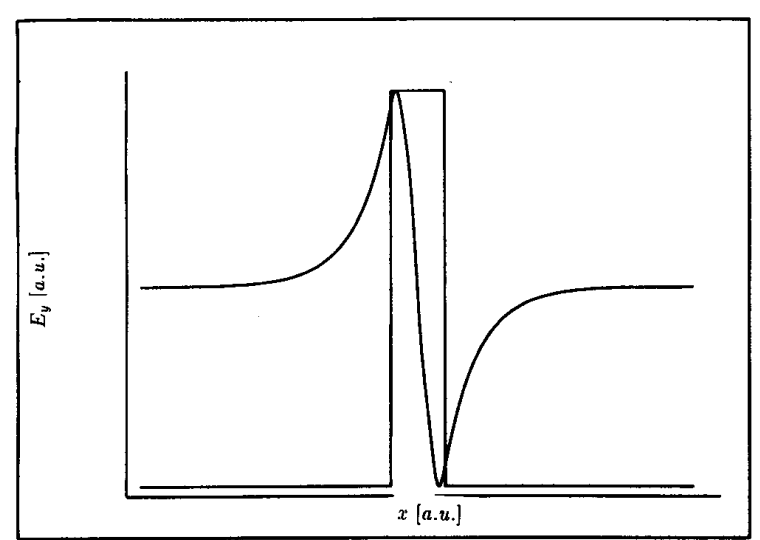

(h)

Fig. 2. (Continued) In (h), the $\mathrm{TE}_{1}$ mode has converged.

TABLE I

\begin{tabular}{|l||l|l|l|l|l|l|}
\hline \multicolumn{1}{|c||}{} & \multicolumn{2}{c|}{ non-absorbing } & \multicolumn{4}{c|}{ absorbing } \\
\hline & new method & slab solver & \multicolumn{2}{c|}{ new method } & \multicolumn{2}{c|}{ slab solver } \\
\hline$T E_{i}$ & $n_{e}$ & $\delta n_{e}$ & $n_{e, r}$ & $n_{e, i}$ & $\delta n_{e, r}$ & $\delta n_{e, i}$ \\
\hline 0 & 1.59755263 & $2.310^{-7}$ & 1.59755259 & $9.15210^{-5}$ & $2.310^{-7}$ & $<10^{-8}$ \\
1 & 1.59146905 & $-1.810^{-7}$ & 1.59146917 & $5.32210^{-5}$ & $-1.810^{-7}$ & $<10^{-8}$ \\
\hline
\end{tabular}

Real $\left(n_{e, r_{j}}\right)$ and imaginary $\left(n_{e, i_{j}}\right)$ part of the effective index $n_{e, r_{j}}-i n_{e, i_{j}}$ for modal fields of structures described in text. Also the values as calculated with a slab solver method are given, in the form $\delta n_{e}=n_{e, s l a b}-n_{e, \text { new }}$. For the nonabsorbing (absorbing) structure, the two successive modes were found after $12+19=31(12+20=32)$ propagation steps.

TABLE II

\begin{tabular}{|l||l|l||l|l|l|l|}
\hline \multicolumn{1}{|c||}{} & \multicolumn{2}{c|}{ non-absorbing } & \multicolumn{4}{c|}{ absorbing } \\
\hline & new method & slab solver & \multicolumn{2}{c|}{ new method } & \multicolumn{2}{c|}{ slab solver } \\
\hline$T E_{i}$ & $n_{e}$ & $\delta n_{e}$ & $n_{e, r}$ & $n_{e, i}$ & $\delta n_{e, r}$ & $\delta n_{e, i}$ \\
\hline 0 & 1.59755302 & $2.310^{-7}$ & 1.59755297 & $9.15010^{-5}$ & $2.310^{-7}$ & $<10^{-8}$ \\
1 & 1.59755225 & $2.310^{-7}$ & 1.59755220 & $9.15410^{-5}$ & $2.310^{-7}$ & $<10^{-8}$ \\
2 & 1.59150570 & $-1.910^{-7}$ & 1.59150539 & $5.18110^{-5}$ & $-1.910^{-7}$ & $<10^{-8}$ \\
3 & 1.59142826 & $-2.010^{-7}$ & 1.59142800 & $5.50510^{-5}$ & $-1.910^{-7}$ & $<10^{-8}$ \\
\hline
\end{tabular}

Real $\left(n_{e, r_{j}}\right)$ and imaginary $\left(n_{e, i_{j}}\right)$ part of the effective index $n_{e, r_{j}}-i n_{e, i_{j}}$ for modal fields of structures described in text. Also the values as calculated with a slab solver method are given, in the form $\delta n_{e}=n_{e, s l a b}-n_{e, \text { new }}$. For the nonabsorbing (absorbing) structure,the four successive modes were found after $12+13+28+21=74(12+13+32+20=77)$ propagation steps, respectively.

field propagates towards another mode than the modes which were already found, since these have been filtered out. If this filtering process is carried out after each propagation step, then every time a "filtered out" mode rises again, its power is diminished. Then the same propagation scheme is followed as for the previous mode, resulting in a converging value for the propagation constant, etc.

If the power present in $\Psi^{0 \prime}$ is smaller than some critical small value, then all guided modes are considered to be found. In case radiation modes were present in $\Psi^{0}$, this condition is not fulfilled however. There are two ways out of this problem. One solution is simply to put a lower bound on the effective index. Another solution is to first propagate the initial field along the real $z$-axis in order to keep all guided modes but to suppress all radiation modes (recall that the structure under consideration is $z$-invariant), and then start our propagation along the complex $z$-axis. Then nearly all of the power in $\Psi^{0}$ would consist of guided modes. It may take a lot of propagation steps, however, in order to loose a significant part of the radiation modes energy. Therefore we choose to put 


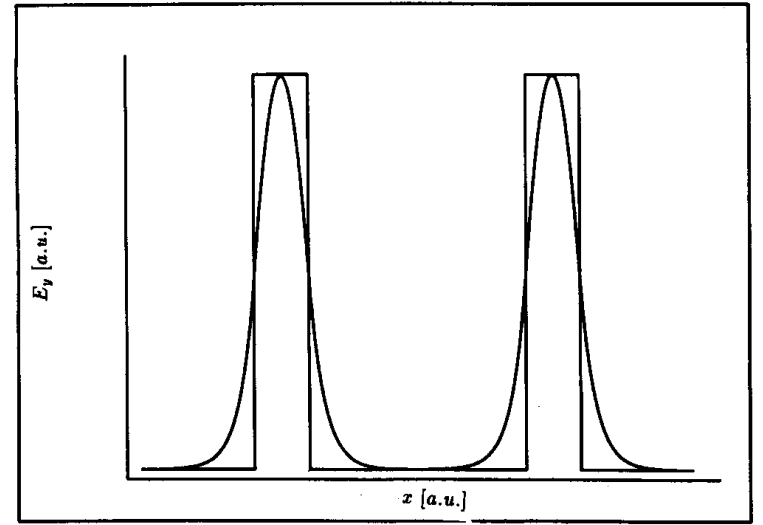

(a)

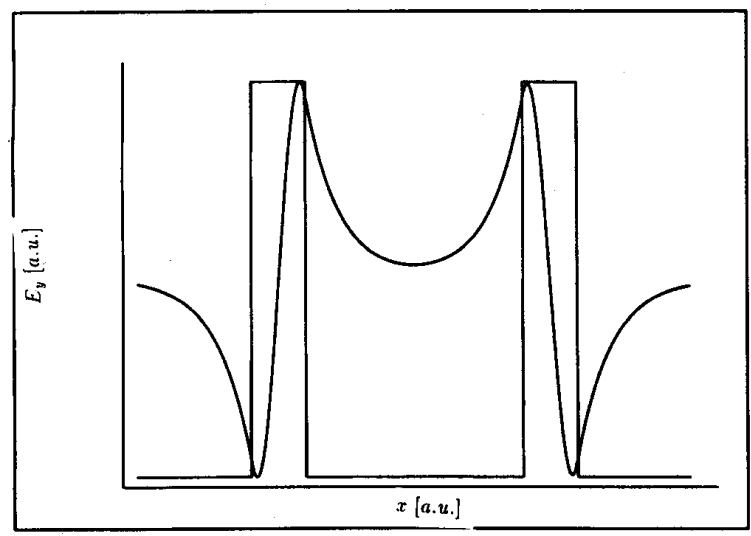

(c)

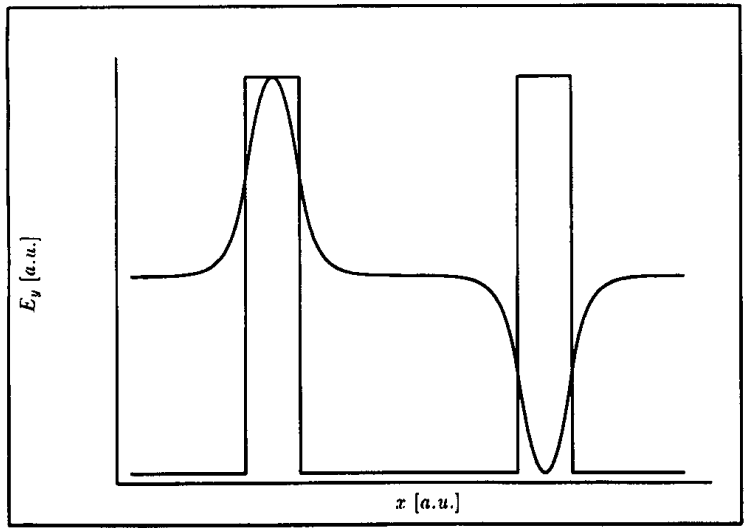

(b)

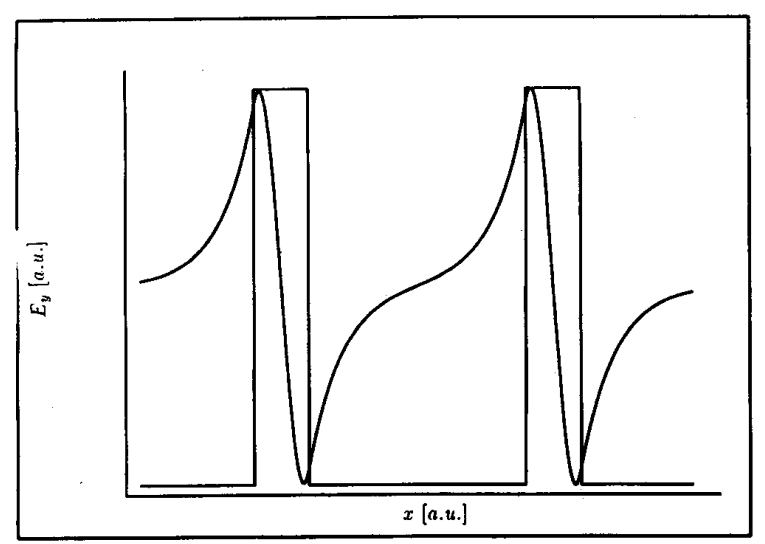

(d)

Fig. 3. Modal fields $\mathrm{TE}_{j}, j=0,1,2,3((\mathrm{a})$-(d)) for the nonabsorbing structure described in text.

a lower bound on the effective index. If for ten successive propagation steps, $n_{e}$ drops below the lower bound, then all guided modes are decided to be found.

Notice that, because the analysis started with (10), in fact a modified version of the inverse iteration method has been used. In the standard inverse iteration method one would solve the matrix equation

$$
\left[M-i \frac{4 \beta_{0}}{\Delta z}\right] \Psi^{s+1}=\Psi^{s} \equiv f^{s}
$$

instead of (10). Tests showed that the two inverse iteration schemes give on the average the same results. Since (10) can be used both as starting equation for our inverse iteration scheme and as propagation equation, we have chosen for convenience of implementation to use the inverse iteration scheme starting with (10) instead of (17).

\section{RESULTS}

In this section some tests for nonabsorbing and absorbing one-dimensional refractive index profiles are presented. In all examples, $n_{0}=1.59$, the wavelength $\lambda_{0}=2 \pi / k_{0}=1.32 \mu \mathrm{m}$, the spacing between successive transverse grid points $\Delta x=$ $0.050 \mu \mathrm{m}$ and the number of transverse grid points is 1024 .
In all tests, the convergence criterion was that six successive values of $\beta_{e_{j}}$ should have a difference less than $10^{-12}$.

- Test 1: In the first test, a $5 \mu \mathrm{m}$ wide symmetric straight waveguide is considered with refractive index $n=1.60$, surrounded by a medium of index $n=1.59$. Fig. 2 shows some successive propagation steps for this structure. As starting field was used a superposition of a number of sine and cosine functions. Within 31 propagation steps, the two constituent modal fields are found. In Table I the effective indexes of the $\mathrm{TE}_{0}$ and $\mathrm{TE}_{1}$ mode, achieved with our method, are compared with the results obtained with a standard slab solver based on the transfer matrix method. The results for a guiding structure of refractive index $n=1.60-i 10^{-4}$, with a surrounding nonabsorbing medium with $n=1.59$, are also given in Table I.

The field correlation factor for $\mathrm{TE}_{0}$ andTE $\mathrm{T}_{1}$, defined by the quantity $\left|<\Theta_{j}, \epsilon_{j}>\right|^{2} /\left|<\epsilon_{j}, \epsilon_{j}>\right|^{2}\left(\boldsymbol{\Theta}_{j}\right.$ and $\epsilon_{j}$ being the modal fields obtained by the present method and the slab solver, respectively), are $1-10^{-11}$ and $1-210^{-10}$, respectively.

- Test 2: In the second test the refractive index profile is the same as in the first test, except that now there two identical $5-\mu \mathrm{m}$-wide waveguides, separated by $11 \mu \mathrm{m}$. Each 
TABLE III

\begin{tabular}{|c|c|c|c|c|c|c|}
\hline & \multicolumn{2}{|c|}{ non-absorbing } & \multicolumn{4}{|c|}{ absorbing } \\
\hline \multirow[b]{2}{*}{$T E_{i}$} & new method & slab solver & \multicolumn{2}{|c|}{ new method } & \multicolumn{2}{|c|}{ slab solver } \\
\hline & $n_{e}$ & $\delta n_{e}$ & $n_{e, r}$ & $n_{e, i}$ & $\delta n_{e, r}$ & $\delta n_{e, i}$ \\
\hline 0 & 1.5975526330 & $2.410^{-7}$ & 1.5975525877 & $9.15197810^{-5}$ & $2.310^{-7}$ & $<10^{-8}$ \\
\hline 1 & 1.5975526320 & $2.410^{-7}$ & 1.5975525867 & $9.15198710^{-5}$ & $2.310^{-7}$ & $<10^{-8}$ \\
\hline 2 & 1.5914708489 & $-8 \quad 10^{-8}$ & 1.5914707 .531 & $5.3163710^{-5}$ & $-1.710^{-7}$ & $-1.310^{-7}$ \\
\hline 3 & 1.5914669541 & $-1.610^{-7}$ & 1.5914666658 & $5.3439910^{-5}$ & $-1.710^{-7}$ & $-210^{-8}$ \\
\hline
\end{tabular}

Real $\left(n_{e, r_{j}}\right)$ and imaginary $\left(n_{e, i_{j}}\right)$ part of the effective index $n_{e, r_{j}}-i n_{e, i_{j}}$ for modal fields of structures described in text. Also the values as calculated with a slab solver method are given, in the form $\delta n_{e}=n_{e, \text { slab }}-n_{e, \text { new }}$. For the nonabsorbing (absorbing) structure, the four successive modes were found after $12+14+43+20=89(12+14+35+20=81)$ propagation steps.

of the effective indexes of test 1 splits into two values. The results are shown in Table II.

- Test 3: In order to investigate the resolution of our method, the two waveguides are separated by $20 \mu \mathrm{m}$. The values for the effective index are shown in Table III.

The four modal field profiles for the nonabsorbing case, obtained by the present method, are displayed in Fig. 3.

The difference between the values obtained with the present method and the exact values are due to the discretization in the transversal direction. With respect to the coupling length in case of the coupled waveguides (Tables II, III), which is inversely proportional to $n_{e_{0}}-n_{e_{1}}$ and $n_{e_{2}}-n_{e_{3}}$, there is nice agreement however between the two methods. As is well known, the computation time grows linearly as function of the number of grid points for the FDBPM. A considerable increase in computer speed can be achieved by first using a coarse grid, and once a mode nearly has been found, to use a finer mesh [21]. This method has not yet been implemented. For two-dimensional refractive index profiles, this option is very interesting, since then the number of grid points can become quite large.

\section{CONCLUSIONS}

A modal fields calculation method has been applied to the Finite Difference BPM scheme, both for nonabsorbing and for absorbing structures. The examples for one-dimensional refractive index structures have shown that the method works well. All guided modes can be found in a reasonably small number of propagation steps. The third example showed that also nearly degenerate modes can be found. It is expected that for two-dimensional refractive index profiles, this method is quite faster than already existing modal fields calculation methods.

\section{ACKNOWLEDGMENT}

It is a pleasure to thank A. Bakker for fruitful discussions and for the big help in the software support. Also useful comments by M.K. Smit are acknowledged.

\section{REFERENCES}

[1] J. van Roey, J. van der Donk, and P. E. Lagasse, "Beam-propagation method: Analysis and assessment," J. Opt. Soc. Am., vol. 71, no. 7, pp. $423-429,1981$

[2] L. Thylen, "The beam propagation method: An analysis of its applicability," Opt. Quantum Electron., vol. 15, pp. 433-439, 1983.

[3] P. E. Lagasse and R. Baets, "Application of propagation beam methods to electromagnetic and acoustic wave propagation problems: A review," Radio Sci., vol. 22, no. 7, pp. 1225-1233, 1987.

[4] D. Yevick and B. Hermansson, "Efficient beam propagation techniques," IEEE J. Quantum Electron., vol. 26, pp. 109-112, 1990.

[5] Y. Chung and N. Dagli, "An assessment of finite difference beam propagation method," IEEE J. Quantum Electron., vol. 26, no. 8, pp: 1335-1339, 1990.

[6] R. Accornero, M. Artiglia, G. Coppa, P. di Vita, G. Lapenta, M. Potenza, and P. Ravetto, "Finite difference methods for the analysis of integrated optical waveguides,” Electron. Lett., vol. 26, no. 23, pp. 1959-1960, 1990.

[7] A. Ghatak, K. Thyagarajan, and M. R. Shenoy, "Numerical analysis of planar optical waveguides using matrix approach," J. Lightwave Technol., vol. LT-5, no. 5, pp. 660-667, 1987.

[8] J. Chilwell and I. Hodgkinson, "Thin-films field-transfer matrix theory of planar multilayer waveguides and reflection from prism-loaded waveguides," J. Opt. Soc. Am. A, vol. 1, no. 7, pp. 742-753, 1984.

[9] K.-H. Schlereth and M. Tacke, "The complex propagation constant of multilayer waveguides: An algorithm for a personal computer," IEEE J. Quantum Electron., vol. 26, no. 4, pp. 627-630, 1990.

[10] R. E. Smith, S. N. Houde-Walter, and G. W. Forbes, "Numerical determination of planar waveguide modes using the analyticity of the dispersion relation," Opt. Lett., vol. 16, no. 17, pp. 1316-1318, 1991.

[11] P. C. Lee, D. Schulz, and E. Voges, "Three-dimensional finite difference beam propagation algorithms for photonic devices," J. Lightwave Technol., vol. 10, no. 12, pp. 1832-1838, 1992.

[12] D. Yevick and B. Hermansson, "New formulations of the matrix beam propagation method: Application to rib waveguides," IEEE J. Quantum Electron., vol. 25, pp. 221-229, 1989.

[13] W. P. Huang, C. L. Xu, S. T. Chu, and S.K. Chaudhuri, "A vector beam propagation method for guided-wave optics," IEEE Photon. Technol. Lett., vol. 3, no. 10, pp. 910-913, 1991.

[14] H. J. W. M. Hoekstra, G. J. M. Krijnen, and P. V. Lambeck, "Efficient interface conditions for the finite difference beam propagation method," J. Lightwave Technol., vol. 10, no. 10, pp. 1352-1355, 1992.

[15] _ _ "On the accuracy of the finite difference method for applications in beam propagating techniques," J. Opt. Commun., vol. 94, pp. 506-508, 1992.

[16] A. Behie and P. K. W. Vinsome, "Block iterative methods for fully implicit reservoir simulation," Soc. Pet. Eng. J., pp. 658-668, Oct. 1982.

[17] W. H. Press, B. P. Flannery, S. A. Teukolsky, and W. T. Vetterling, Numerical Recipes. Cambridge, MA: Cambridge University Press, 1986.

[18] A. R. Mitchell and D. F. Griffiths, The Finite Difference Method in Partial Differential Equations. Chichester: Wiley, 1980

[19] F. Wijnands, T. Rasmussen, H. J. W. M. Hoekstra, J. H. Povlsen, A. Bjarklev, and R. M. de Ridder, to be submitted. 
[20] G. R. Hadley, "Transparent boundary condition for beam propagation," Opt. Lett., vol. 16, no. 9, pp. 624-626, 1991.

[21] T. Rasmussen, J. H. Povlsen, and A. Bjarklev, "Accurate finite difference beam propagation method for complex integrated optical structures," IEEE Photon. Technol. Lett., vol. 5, no. 3, pp. 339-342, 1993.

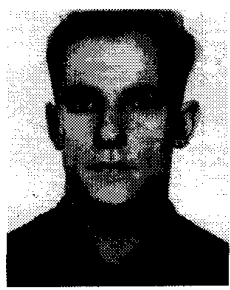

Frank Wijnands received the M.Sc. degree in 1988 and the Ph.D. degree in 1992 both from the University of Nijmegen, The Netherlands, on electronic and crystallographic properties of quasiperiodic crystals.

In 1993 he joined the Lightwave Devices Group at the MESA Research Institute, University of Twente, Enschede, The Netherlands, where he works on mathematical principles and numerical implementation of beam propagation methods in linear and nonlinear integrated optics.

Hugo J. W. M. Hoekstra recieved the M.Sc. degree in experimental physics from the University of Amsterdam in 1977. After being a teacher in high school, he began a Ph.D. study on magneto optical properties of solid transition-metal halides at the University of Groningen where he received the Ph.D. degree in 1984

Between 1984 and 1988 he was a Postdoctoral Fellow in the field of surface science at the Universities of Nijmegen and Groningen. In 1988 he joined the Lightwave Devices Group of the MESA Research Institute at the University of Twente, Enschede, The Netherlands, where he is engaged in work on linear and nonlinear integrated optics.

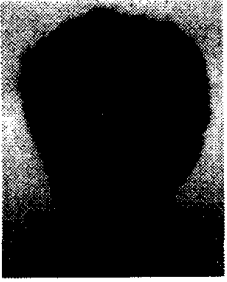

Gijs J. M. Krijnen (S'90-M'93) started his Ph.D. work in the Lightwave Devices Group of the MESA research institute at the University of Twente, Enschede, The Netherlands, in 1987, receiving the M.Sc. degree in the field of magnetic recording at the Philips Laboratories, Eindhoven, The Netherlands. His Ph.D. research concerned integrated nonlinear optical switching devices.

After finishing his Ph.D. work in May 1992 he started work as a Postdoctoral Fellow of the Roya Netherlands Academy of Arts and Sciences. In this current work his main interest is in the field of the application of nonlinear integrated optics devices as sensors.

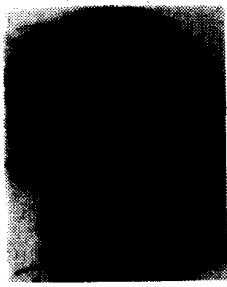

René M. de Ridder was born April 26, 1950 in Amsterdam, The Netherlands. He studied electrical engineering at the University of Twente, Enschede, The Netherlands, where he received the masters degree in 1978 and the Ph.D. degree in 1988

Since 1981 he has been employed as a lecturer and research scientist at the University of Twente, where he is now a member of the Lightwave Devices Group in the MESA Research Institute. His current research interests include integrated-optic devices for optical communications. 